\title{
Diminishing Apoptosis by Deletion of Bax or Overexpression of Bcl-2 Does Not Protect against Infectious Prion Toxicity In Vivo
}

\author{
Andrew D. Steele, ${ }^{1}$ Oliver D. King, ${ }^{1}$ Walker S. Jackson, ${ }^{1}$ Claudio A. Hetz, ${ }^{2,3}$ Andrew W. Borkowski, ${ }^{1}$ Peter Thielen, ${ }^{2}$ \\ Robert Wollmann, ${ }^{4}$ and Susan Lindquist ${ }^{1}$ \\ ${ }^{1}$ Whitehead Institute for Biomedical Research, Howard Hughes Medical Institute, Massachusetts Institute of Technology, Cambridge, Massachusetts 02142, \\ ${ }^{2}$ Department of Immunology and Infectious Diseases, Harvard School of Public Health, Boston, Massachusetts 02115, ${ }^{3}$ Department of Cellular and \\ Molecular Biology, Institute of Biomedical Sciences, Fondo Nacional Areas Prioritas Center for Molecular Studies of the Cell, University of Chile, Santiago, \\ Chile, and ${ }^{4}$ Department of Pathology, University of Chicago, Chicago, Illinois 60637
}

B-cell lymphoma protein $2(\mathrm{Bcl}-2)$ and $\mathrm{Bcl}$-2-associated $\mathrm{X}$ protein (Bax), key antiapoptotic and proapoptotic proteins, respectively, have important roles in acute and chronic models of neurologic disease. Several studies have implicated Bax and Bcl-2 in mediating neurotoxicity in prion diseases. To determine whether diminishing apoptotic cell death is protective in an infectious prion disease model we inoculated mice that either were null for proapoptotic Bax or overexpressed antiapoptotic Bcl-2. Interestingly, genetic manipulation of apoptosis did not lessen the clinical severity of disease. Moreover, some disease parameters, such as behavioral alterations and death, occurred slightly earlier in mice that are null for Bax or overexpress Bcl-2. These results suggest that Bax and Bcl-2 mediated apoptotic pathways are not the major contributing factor to the clinical or pathological features of infectious prion disease.

Key words: PrP; home cage; amyloid; cell death; necrosis; transmissible

\section{Introduction}

The pathological events observed in neurodegenerative disease culminate in a dramatic loss of neurons in the brain but the importance of cell death pathways in this process is controversial (Yuan et al., 2003; Shacka and Roth, 2006; Steele and Yi, 2006). Genetic manipulations of proapoptotic or antiapoptotic proteins have, in many cases, ameliorated pathological or clinical features in mouse models of neurodegenerative disease, including amyotrophic lateral sclerosis, ischemia, and axotomy (Dubois-Dauphin et al., 1994; Martinou et al., 1994; Kostic et al., 1997; Gould et al., 2006; Tsai et al., 2006; Li et al., 2007). However, in other studies modification of apoptosis has not altered the symptoms of neurodegeneration (Sagot et al., 1995; Selimi et al., 2000; Kang et al., 2003; Chiesa et al., 2005; Coulpier et al., 2006). Thus, apoptotic pathways have significant disease-modifying effects in a subset of neurodegenerative diseases.

Prion diseases $(\mathrm{PrD})$ are a unique class of neurodegenerative disorders manifesting in genetic, sporadic, and infectious forms, affecting humans as well as wild and domesticated animals

Received July 19, 2007; revised Sept. 11, 2007; accepted 0ct. 13, 2007.

This work was supported by U.S. Department of Defense Grant DAMD17-00-1-0296 and the Howard Hughes Medical Institute (S.L.) and FONDECYT Grant 1070444, FONDAP Grant 15010006, and High Q Foundation (C.H.). We are grateful to Artur Topolszki for expert technical assistance, to Jill McCutcheon and Nancy Forger (University of Massachusetts, Boston, MA) for providing us with the $\mathrm{BCl}-2$ over-expression mice, and to Karen Allendoerfer and other members of the Lindquist laboratory for critical comments on this manuscript.

Correspondence should be addressed to Susan Lindquist, Whitehead Institute for Biomedical Research, 9 Cambridge Center, Cambridge, MA 02142. E-mail: Lindquist_admin@wi.mit.edu.

DOI:10.1523/JNEUROSCI.3290-07.2007

Copyright $\odot 2007$ Society for Neuroscience $\quad$ 0270-6474/07/2713022-06\$15.00/0
(Prusiner, 1998; Aguzzi and Heikenwalder, 2006; Caughey and Baron, 2006). In infectious forms of the disease, the normal cellular isoform of the prion protein, $\operatorname{PrP}$ (termed $\operatorname{PrP}^{\mathrm{C}}$ ), is structurally converted into $\mathrm{PrP}^{\mathrm{Sc}}$, a self-perpetuating and aggregationprone conformation of the protein (Prusiner, 1998). The ongoing conversion of $\operatorname{PrP}^{\mathrm{C}}$ to $\operatorname{PrP}^{\mathrm{Sc}}$ is required for prion toxicity; however, beyond this observation little is known about the mechanism by which this process triggers neurotoxicity (Aguzzi and Heikenwalder, 2006).

Prion infections cause characteristic lesions in the brain: spongiform vacuolation, aggregates of PrP, activation of microglia, proliferation of astrocytes, and ultimately loss of neurons (Aguzzi and Heikenwalder, 2006). The mechanism(s) of neuronal loss is unknown, but various forms of neuronal death, including apoptosis, have been documented in diverse hosts with PrD (Liberski et al., 2004). Apoptosis has been described in the brains of patients affected by Creutzfeldt-Jakob disease and fatal familial insomnia and in prion-infected hamsters, mice, and sheep (Liberski et al., 2004). Several studies (discussed below) have suggested important roles for B-cell lymphoma protein 2 (Bcl-2)associated $\mathrm{X}$ protein (Bax) and $\mathrm{Bcl}-2$ in the neurotoxic pathway caused by PrP mutants (Chiesa et al., 2005; Li et al., 2007; Nicolas et al., 2007).

Bax and Bcl-2 are at the core of the apoptotic pathway located at the mitochondria where multiple cell death signals converge to trigger apoptosis (Yuan et al., 2003). Downstream of apoptotic stimuli, Bax induces cell death by intramembranous homooligomerization and resultant permeabilization of the mitochondrial outer membrane, leading to release of cytochrome $c$ and activation of caspases (Danial and Korsmeyer, 2004). The Bcl-2 
A.

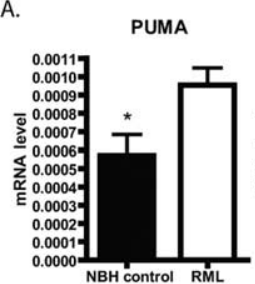$$
\text { B. }
$$

$$
\text { Bim }
$$

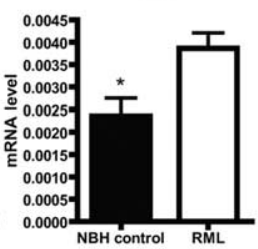

C.

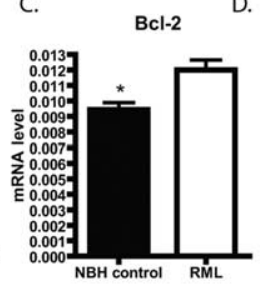

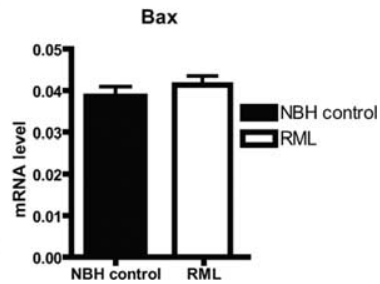

Figure 1. Alterations in the expression of proapoptotic and antiapoptotic genes in prion disease. RT-PCR was performed on trizol extracted brain samples from CD1 mice between 4 and 4.5 months after infection with prions or normal brain homogenate as a control. $\boldsymbol{A}-\boldsymbol{D}$, Levels of PUMA (A), Bim $(\boldsymbol{B}), B C-2(\boldsymbol{C})$, and $B a x(\boldsymbol{D})$ were examined. ${ }^{*} p<0.05$, Student's $t$ test.

family is comprised of proapoptotic and antiapoptotic members, and is defined by the presence of up to four conserved domains within their primary structure (Reed, 2006). Proapoptotic members can be further subdivided into more fully conserved, "multidomain" members containing Bax homology (BH) 1-3 or "BH3-only" members, such as Bim and Puma, which activate Bax.

To address directly the role of apoptosis in infectious $\operatorname{PrD}$, we inoculated Bax knock-out (KO) and $\mathrm{Bcl}-2$ neuronal overexpression transgenic ( $\mathrm{Tg}$ ) mice with the Rocky Mountain Laboratory (RML) strain of murine prions. To our surprise, we observed that $\mathrm{Bax}$ deficient and $\mathrm{Bcl}-2$ overexpression Tg mice were not protected against prion toxicity. In fact, some features of $\mathrm{PrD}$, such as behavioral alterations and survival, were made worse, whereas accumulation of proteinase- $\mathrm{K}$ resistant $\operatorname{PrP}$ and pathological changes were unaltered by diminishing apoptosis.

\section{Materials and Methods}

RNA extraction and quantitative RT-PCR. Total RNA was prepared from brain homogenates using trizol (Invitrogen, Carlsbad, CA) and cDNA was synthesized with SuperScript III (Invitrogen) using random primers. Quantitative real-time (RT)-PCRs using SYBR green fluorescent reagent were performed in an ABI PRISM 7700 (Applied Biosystems, Foster City, CA). Relative amounts of mRNAs were calculated from the values of comparative threshold cycle by using $\beta$ actin as a control. Primer sequences were designed by Primer Express software (Applied Biosystems) and are identical to those reported in (Hetz et al., 2007).

Mouse strains, prion inoculations, and behavioral analysis. All mouse experiments were approved by the Massachusetts Institute of Technology Committee on Animal Care. Food and water were provided ad libitum and mice were group housed ( $n=2-5$ per cage) while being maintained on a $12 \mathrm{~h}$ light/dark cycle. Bax KO mice (Knudson et al., 1995) were obtained from The Jackson Laboratory (Bar Harbor, ME) at N8 generations of backcross to C57BL/6J, and were backcrossed one additional time. All mice used in our studies were generated by intercrossing Bax $+/-$ by Bax $+/-$ mice to generate littermate controls. Bcl-2 Tg mice (Dubois-Dauphin et al., 1994) were kindly provided by Nancy Forger (University of Massachusetts, Amherst, MA). This line was maintained on a mixed background composed of C57BL/6 and DBA/2 and was crossed once to C57BL/6 for ovarian transfer rederivation. For breeding we crossed $\mathrm{Bcl}-2 \mathrm{Tg}+/-$ males to $\mathrm{Tg}-/-$ females. For prion injections, mice were injected intracranially with $30 \mu \mathrm{l}$ of $0.1 \%$ brain homogenate containing $\sim 5.5 \mathrm{log}$ $\mathrm{LD}_{50} / 30 \mu \mathrm{l}$ infectious units of RML murine prions. Behavioral analysis was performed essentially as described by Steele et al. (2007). Briefly, mice were placed in new cages containing minimal bedding for a $24 \mathrm{~h}$ video recording using dim red lighting during the dark cycle. Behavioral analysis of videos was performed using HomecageScan 2.0 with definitions as previously defined with the exception that "remain low" and "sleep" were merged under the title "rest" because the steel coat color in some of the Bax KO mice made it difficult to distinguish these two classes of inactivity.

Proteinase-K treatment and Western blotting. Ten percent homogenates (weight/volume) of whole brain were made in PBS from tissue frozen at 4.5 and 5.0 months post inoculation (mpi). Tissue was homogenized in a glass dounce homogenizer and sonicated, and then large debris were pelleted by low speed centrifugation ( $\sim 500 \mathrm{~g}$ for $5 \mathrm{~min})$. Homogenates were diluted to $1 \%$ in lysis buffer consisting of PBS and $1 \%$ Triton $\mathrm{X}-100$ and $1 \%$ Tween 20 and treated with $50 \mu \mathrm{g} / \mathrm{ml}$ proteinase-K (PK) for $1 \mathrm{~h}$ at 37C, followed by boiling in NuPAGE lithium dodecyl sulfate running buffer (Invitrogen) and electrophoresed in NuPAGE Novex (Wadworth, OH) Bis-Tris midi gels (Invitrogen). Proteins were transferred to nitrocellulose membranes which were blocked using 5\% milk and probed with SAF83 (Cayman Chemical, Ann Arbor, MI) to detect PrP and an antibody against $\beta$-tubulin was used for a loading control. A Licor Odyssey was used to detect infrared conjugated secondary antibodies (Rockland, Gilbertsville, PA).

Neuorpathological analysis. Brains were immersion fixed in formalin, paraffin embedded, and sectioned coronally. Five micron sections were stained with hematoxylin and eosin. For glial fibrillary acidic protein (GFAP) immunostaining, five micron thick paraffin sections were deparaffinized and then incubated in 98.6 degree waterbath at pH 6.0 for $40 \mathrm{~min}$, after which the sections were stained using an automated stainer, using an rabbit anti-cow GFAP antibody at 1:400 dilution (Dako, High Wycombe, UK). A blinded analysis of vacuolation and gliosis in the hippocampus, thalamus, striatum, cortex, and cerebellum was conducted. Terminal deoxynucleotidyl transferasemediated biotinylated UTP nick end labeling (TUNEL) staining was performed according to the manufacturer's protocol (Roche, Welwyn Garden City, UK).

Statistical analysis. Statistical analysis was performed using GraphPad Prism. Student's $t$ test was used for analysis of RT-PCR data, log rank test was used for survival data, and Wilcoxon rank sum test (nonparametric) was used for behavioral analysis.

\section{Results}

\section{Expression levels of Bcl-2 family members}

Alterations in the expression levels of proapoptotic and antiapoptotic proteins can be indicative of activation of particular cell death pathways. Therefore, we examined the expression levels of several key proapoptotic and antiapoptotic genes in the brains of RML infected CD1 mice that were killed between 4 and 4.5 months after infection, which is $\sim 3-4$ weeks before the mice become moribund. By quantitative RT-PCR, we observed a significant increase in the RNA levels of Puma and Bim, two potent activators of Bax-dependent apoptosis, when compared with control mice injected with normal brain homogenate ( $p<0.05$, Student's $t$ test, $n=4$ mice per group; Fig. $1 A, B$ ). In addition, a small but reproducible increase in the mRNA levels of $\mathrm{Bcl}-2$ was observed in prion infected mice, possibly reflecting a compensatory effect (Fig. 1C). Similar changes in expression of $\mathrm{Bcl}-2$ were observed in mice infected with another strain of prions, 139A (data not shown). We did not observe significant changes in the mRNA levels of Bax (Fig. $1 D$ ) or Bcl- $\mathrm{X}_{\mathrm{L}}$ (data not shown) in RML infected mice. Because similar changes in the expression of these upstream regulators, Bim and Puma, were observed in a mouse model of amyotrophic lateral sclerosis (Hetz et al., 2007) where Bax deletion significantly delayed cell loss and behavioral onset (Gould et al., 2006), our RT-PCR results suggested that prion infection may activate the main proapoptotic machinery. 
Prion disease in Bax knock-out mice

Because Bax is required for neuronal apoptosis in a variety of disease models (Chiesa et al., 2005; Gould et al., 2006; Heitz et al., 2007; Li et al., 2007) and its deletion delays disease symptoms in several models of neurodegeneration (Gould et al., 2006; Tsai et al., 2006; Li et al., 2007), we hypothesized that Bax KO mice would be protected against prion toxicity. To test this, we inoculated C57BL/6 Bax KO and wild-type (WT) littermate controls with $5.5 \operatorname{logLD}{ }_{50} / 30 \mu$ l of RML prions intracranially (i.c.). We monitored their home cage behaviors using a high resolution automated system which presented robust diagnosis of $\mathrm{PrD}$ in mice in a previous study (Steele et al., 2007). After disease onset, RML infected mice show a striking increase in physical activity as manifested by increases in behaviors such as walking and jumping; other behaviors, such as grooming and hanging decrease during later stages of PrD. We video recorded and analyzed prion-infected Bax KOs $(n=13-$ $16)$ and WT littermates $(n=14-18)$ twice monthly beginning at 3 months postinoculation (mpi), which is just before onset of symptoms in RML-infected mice (Steele et al., 2007).

The overall profile of behavioral changes was similar in prion-infected Bax $\mathrm{KO}$ and control mice. However, several behavioral changes previously shown to be altered in RML PrD in C57BL/6 mice were observed in the Bax KO mice before their WT littermate controls, suggesting that some PrD symptoms have an earlier onset in Bax KO mice. The prion-inoculated Bax KOs had a subtle increase in "walking" over controls at 3.0 and $3.5 \mathrm{mpi}$ (Fig. 2A), a subtle decrease in "resting" at 3.0 and 3.5 mpi (Fig. 2B), no change in grooming (Fig. $2 C$ ), and a small decrease in "hang vertical" at $4.0 \mathrm{mpi}$ (Fig. $2 \mathrm{D}$ ). Interestingly, later in disease progression two behaviors, "walk" and "rest," were also significantly altered between $\operatorname{Bax} \mathrm{KO}$ and control mice. Importantly, the behavior of uninoculated Bax KO mice was indistinguishable from uninoculated WT controls with the exception of "eating," which was slightly elevated in Bax KO mice (data not shown).

Given that the behavioral onset in prion-infected Bax KOs was subtly enhanced, we next measured the survival and pathology of these mice after prion inoculation. We observed a $7 \mathrm{~d}$ enhancement of disease in Bax $\mathrm{KO}$ mice in terms of median survival (median survival for Bax WT, $n=23$, was $181 \mathrm{dpi}$, and for Bax $\mathrm{KO}, n=13$, was $174 \mathrm{dpi} ; p=0.041$ ) (Fig. $2 E$ ). This result suggests that deletion of Bax causes a very subtle enhancement of disease. Next, we inspected brains from prion-infected Bax KO and control mice at 4.5 and $5 \mathrm{mpi}, \sim 1-1.5$ months before morbidity.
A.

C.

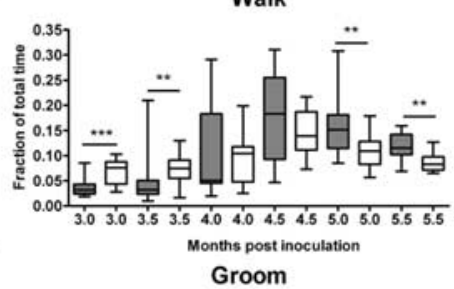

E.
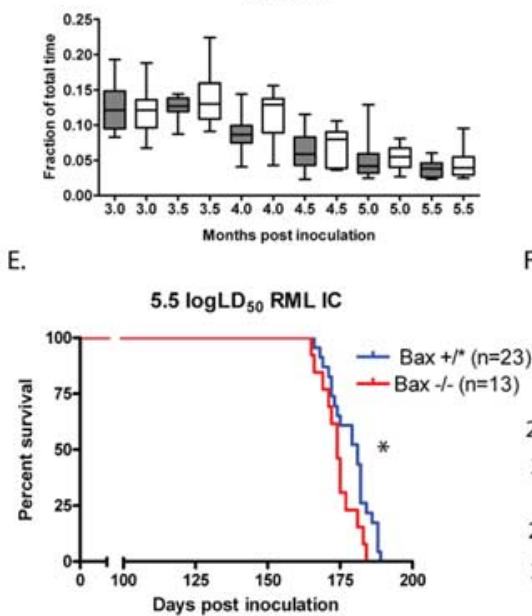

B. Rest

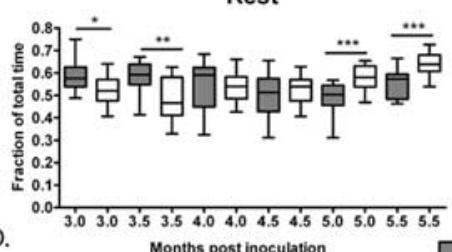

D.
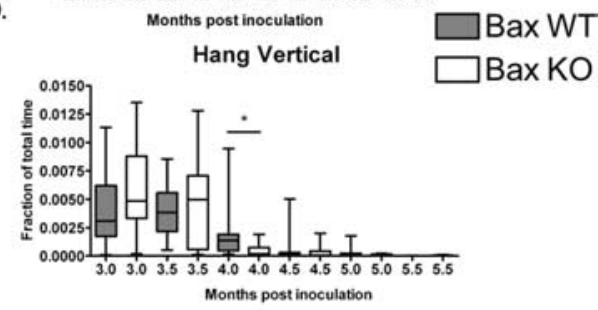

F.

Time: $\begin{array}{llllllll}4.5 & 4.5 & 4.5 & 4.5 & 5 & 5 & 5 & 5 \\ \end{array}$
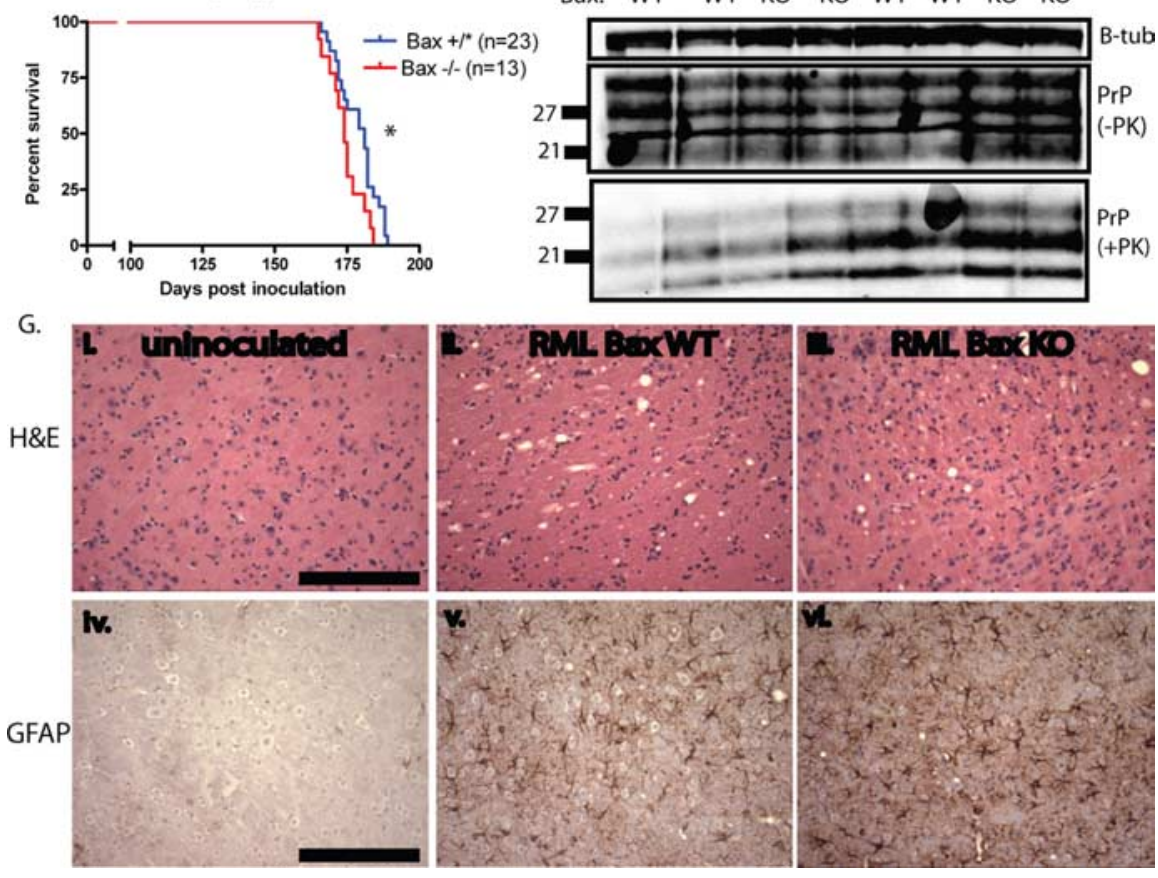

Figure 2. Deletion of $B a x$ does not protect against prion disease. $A-D, B$ ox-and-whisker plots for behaviors known to be altered in prion disease such as walk $(\boldsymbol{A})$, rest $(\boldsymbol{B})$, groom $(\boldsymbol{C})$, and hang vertical $(\boldsymbol{D})$ are shown as for Bax knock-out (white bars) and controls (gray bars). Sample sizes were $n=13-16$ at each time point for Bax KOs and $n=14-18$ for WT controls. Statistically significant results are denoted with asterisks $\left({ }^{*} p<0.05 ;{ }^{* *} p<0.01 ;{ }^{* * *} p<0.001\right)$ and were computed using a Wilcoxon rank sum test. The top and bottom whiskers represent the maximum and minimum values for each time point whereas the box represents the 25 th to 75 th percentiles, and the line represents the median value. $\boldsymbol{E}, B a x$ KO mice die faster from prion infection ( $p=0.041$, log rank test). $\boldsymbol{F}$, Accumulation of proteinase-K-resistant PrP was measured at 4.5 and 5.0 mpi by Western blotting $n=2$ mice per genotype per time point. $\mathbf{G}$, The neuropathological changes were similar in prion-infected $B a x K O$ and control mice, shown at $5 \mathrm{mpi}$, in terms of vacuolation in hematoxylinand eosin-stained sections of uninoculated WT (i), prion-inoculated Bax WT (ii), and Bax KO (iii). Gliosis measured by staining for GFAP was performed on sections from uninoculated WT mice, which did not show any staining (iv), and on prion-inoculated Bax WT (v) and Bax KO (vi) samples. Scale bar, $100 \mu \mathrm{m}$.

Mock-inoculated controls of both genotypes did not show any spongiform changes, nor was there any gliosis or evidence for neuronal loss (Fig. 2Gi,iv). Brains from prion-inoculated Bax KO and WT controls showed dramatic vacuolation visualized using a hematoxylin and eosin stain (Fig. 2Gii,iii). Widespread gliosis, a hallmark of neurodegeneration, was visualized with a stain for GFAP in the brains from prion-infected Bax KO and WT mice (Fig. 2Gv,vi). There were no obvious differences in pathology between Bax KO and controls in the hippocampus, thalamus, striatum, cortex, and cerebellum. To examine cell death in 
A.

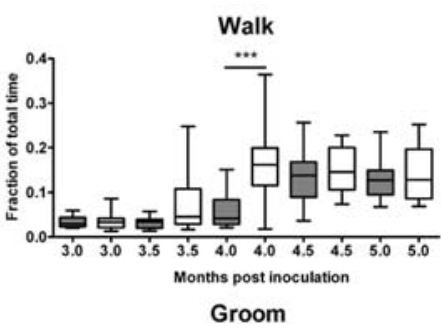

C.

E.
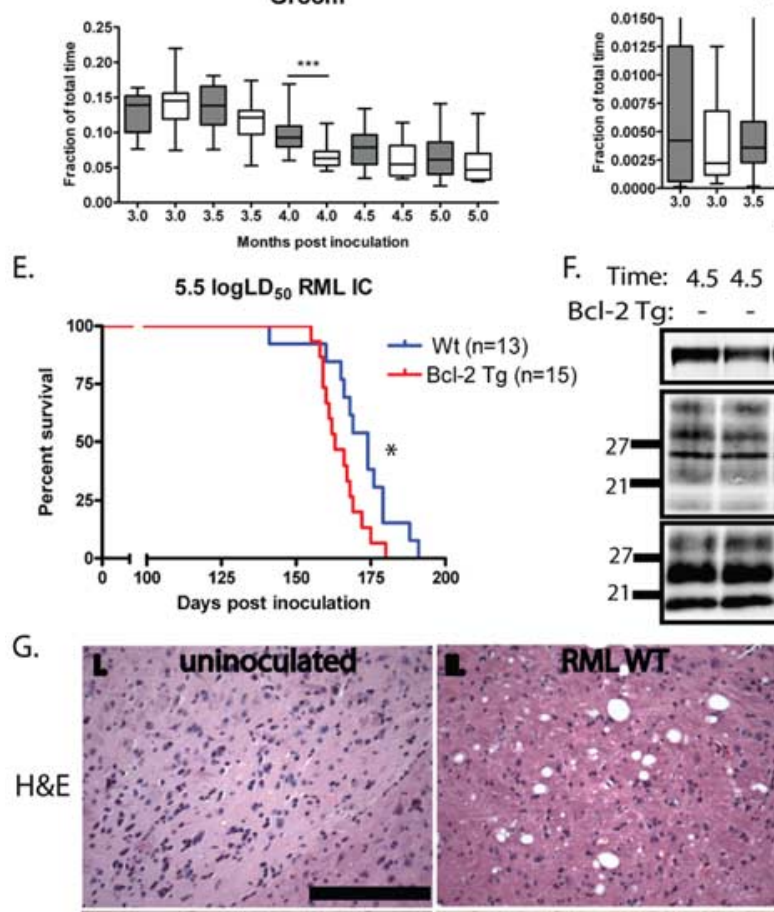

B.
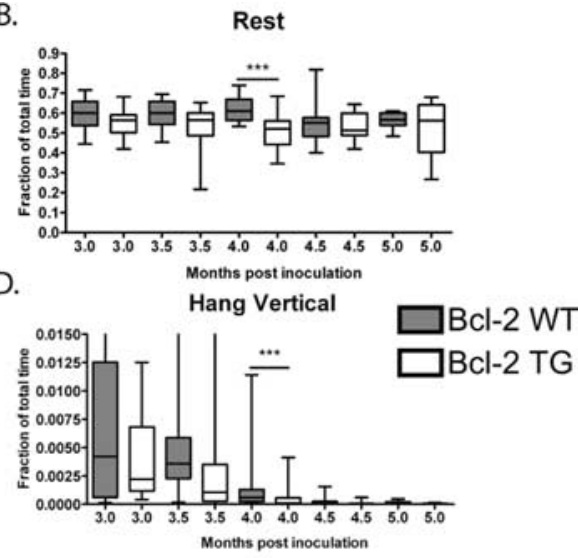

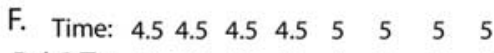
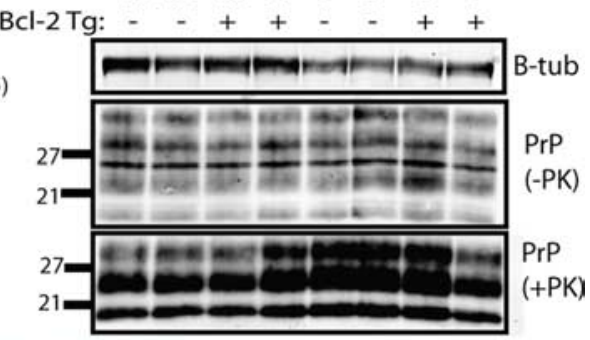

$(+\mathrm{PK})$

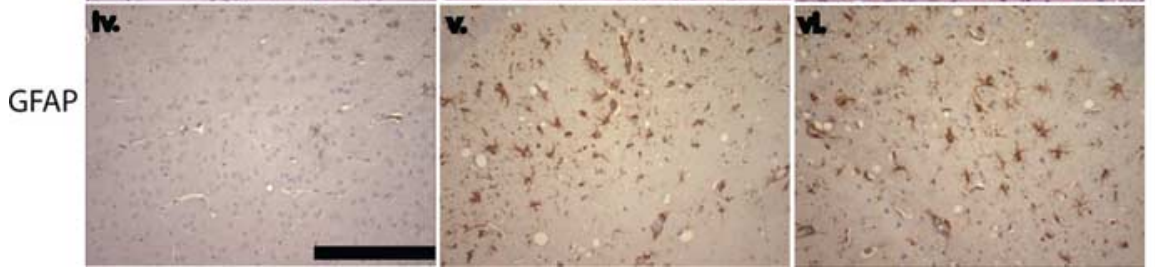

Figure 3. Overexpression of $B C l-2$ in neurons does not protect against prion disease. $A-D, B 0 x$-and-whisker plots for several behaviors known to be altered in prion disease such as walk $(\boldsymbol{A})$, rest $(\boldsymbol{B})$, groom $(\boldsymbol{C})$, and hang vertical $(\boldsymbol{D})$ are shown as for $B C l-2$ TG (gray bars) and controls (white bars). Statistically significant results are denoted with asterisks ( ${ }^{*} p<0.05$; ${ }^{* *} p<0.01$; ${ }^{* * *} p<0.001$ ) and were computed using a Wilcoxon rank sum test. $E, B C l-2$ Tg mice die faster from prion infection ( $p=0.03, \log$ rank test. $\boldsymbol{F}$, Accumulation of proteinase-K resistant PrP was measured at 4.5 and 5.0 mpi by Western blotting $n=2$ mice per genotype per time point. $\mathbf{G}$, The neuropathological changes were similar in prion-infected $B C-2 \mathrm{Tg}$ and control mice, shown at 5 mpi, in terms of vacuolation in hematoxylin- and eosin-stained sections of uninoculated WT (i), prion-inoculated WT (ii) and BCl-2 $\mathrm{Tg}$ (iiii). Gliosis measured by staining for GFAP was performed on sections from uninoculated WT mice which did not show any staining (iv) and on prion-inoculated WT (v) and BCl-2 Tg (vi) samples. Scale bar, $100 \mu \mathrm{m}$.

in the brain. Digestion with PK suggested that $\operatorname{Bax} \mathrm{KO}(n=2)$ and WT controls $(n=$ 2) had similar amounts of prion deposition at the time points examined (Fig. $2 F$ ).

\section{Prion disease in Bcl-2 neuronal} overexpression transgenic mice

Because deleting Bax had a surprising and subtle enhancing effect on prion disease, we tested another mouse model with diminished apoptosis. Bcl-2 has a broad antiapoptotic function and its overexpression protects neurons from damage and death in a variety of models (DuboisDauphin et al., 1994; Martinou et al., 1994; Chen et al., 1997; Kostic et al., 1997; Nicolas et al., 2007). We obtained mice (C57BL/6, DBA/2 hybrids) that specifically overexpress human Bcl-2 in neurons under the neuronal enolase promote (Martinou et al., 1994). We inoculated Bcl-2 Tgs and WT littermate controls with $5.5 \operatorname{logLD}{ }_{50} / 30 \mu \mathrm{l}$ RL prions (i.c.) and analyzed their behavior twice monthly beginning at $3 \mathrm{mpi}$. Similar to what we observed in the Bax KOs infected with prions, several known PrD-related behavioral alterations occurred earlier in the $\mathrm{Bcl}-2 \mathrm{Tg}$ mice compared with controls. For example, an increase in "walk" occurred earlier in Bcl-2 Tgs at $4.0 \mathrm{mpi}$ (Fig. 3A), and "rest" (Fig. 3B), "groom" (Fig. 3C), and "hang vertical” (Fig. 3D) all showed decreases first in $\mathrm{Bcl}-2$ Tgs at $4.0 \mathrm{mpi}$.

The $B c l-2$ Tgs also succumbed to $\operatorname{PrD}$ slightly faster than the WT controls; the median survival of $\mathrm{Bcl}-2 \mathrm{Tgs}$ was $11 \mathrm{~d}$ shorter than that of controls (the median survival for WT controls was $174 \mathrm{dpi}$, whereas for $\mathrm{Bcl}-2$ Tgs the median survival was $163 \mathrm{dpi} ; p=0.030$, log rank test) (Fig. 3E). Thus, in this model of $\operatorname{PrD}$, too, there is a subtle enhancement of incubation time, suggesting that Bcl-2 over-expression does not protect against prion toxicity and may even cause a very subtle enhancement of infectious PrD.

At the neuropathological level, brains from prion-infected $\mathrm{Bcl}-2$ Tgs and control mice at 4.5 and 5 mpi showed similar dramatic vacuolation (Fig. 3Gii, iii) and gliosis

PrD brains, we performed TUNEL staining on samples taken at 4.5 and 5.0 mpi. The brains of Bax WT and Bax KO were almost completely devoid of TUNEL-positive cells with the exception of the granular layer of the cerebellum, which showed an equivalent amount of TUNEL staining between both genotypes (and no staining in mock inoculated controls) (data not shown).

Finally, we assayed the formation of PK-resistant PrP, the classic surrogate marker for $\operatorname{PrD}$, by treating brain homogenates from Bax KO and control mice ( $n=2$ per group) at 4.5 and 5.0 mpi with PK. PK-resistant PrP is the classic surrogate marker for the buildup of prion infectivity and the amount of aggregated PrP in all regions examined (Fig. $3 G v, v i$ ). There were no obvious differences in pathology in the hippocampus, thalamus, striatum, cortex, and cerebellum. We also assayed the amount of proteinase- $\mathrm{K}$ resistant $\mathrm{PrP}$ by treating brain homogenates from $B c l-2$ Tgs and controls at 4.5 and $5 \mathrm{mpi}$ with $50 \mu \mathrm{g} / \mathrm{ml} \mathrm{PK}$; no differences were noted ( $n=2$ mice per group per time point) (Fig. 3F).

\section{Discussion}

By RT-PCR we observed small alterations in the expression of proapoptotic and antiapoptotic genes in prion-inoculated mice before overt illness, consistent with other reports (Park 
et al., 2000; Lyahyai et al., 2006). These results suggested that apoptotic pathways could be involved in mediating prioninduced neurodegeneration. Accordingly, we challenged two well characterized mouse lines with demonstrated resistance to apoptosis with RML prions. Surprisingly both deletion of Bax and overexpression of Bcl-2 had subtle enhancing effects on PrD.

The role of apoptosis in genetic forms of PrD has been studied in several systems, both in vitro and in vivo. Apoptotic cell death has been observed in primary cultured neurons infected with prions (Cronier et al., 2004), treated with proteasome inhibitors (Ma et al., 2002), or both (Kristiansen et al., 2005). In a $\mathrm{Tg}$ mouse expressing a repeat expansion of $\operatorname{PrP}$ associated with disease in humans, deletion of Bax did not affect the behavioral phenotype, but rescued the cerebellar granular neurons from death (Chiesa et al., 2005). The complexity of prion-induced death is underscored in a series of previous studies examining the roles of Bax and Bcl-2 in mediating mutant PrP toxicity in vivo. For example, Li and colleagues deleted Bax from two additional mutant PrP Tg models and observed that Bax deletion delayed cell loss in one deletion mutant line (PrP $\Delta 32-134)$ although not affecting cell loss in another deletion mutant line (PrP $\Delta 105-125)$ ( $\mathrm{Li}$ et al., 2007). A previous study demonstrated a protective role of Bcl-2 overexpression in a mutant $\operatorname{PrP} \operatorname{Tg}(\operatorname{PrP} \Delta 32-134)$, as $\mathrm{Bcl}-2$ overexpression delays neuronal loss and extends survival in these Tg mice (Nicolas et al., 2007). Finally, one group determined that the toxicity induced by ectopic expression of the PrP ortholog, doppel (Dpl), in the brain of mice lacking PrP is diminished by deletion of Bax (Heitz et al., 2007) whereas another group failed to detect a rescue after deletion of Bax in a different model of Dpl toxicity (Dong et al., 2007). Thus, Bax-dependent and -independent pathways may mediate the toxicity of ectopic Dpl expression in the CNS.

One study of infectious bovine prions in Bax KO mice did not detect any differences between Bax KOs and controls in terms of survival or pathological changes (Coulpier et al., 2006). Our results are overall quite complementary to this study with the slight discrepancy that we see a small enhancement of disease in terms of the survival of Bax KOs inoculated with RML prions. This could be the result of using different prion strains or simply that the effect that we observed in the Bax KOs is subtle. Because we did not observe protection against cell death in prion-infected $B a x$ KOs and neuronal $\mathrm{Bcl}-2$ overexpression Tgs, the mechanism by which infectious PrP mediates toxicity must differ from that of mutant PrP and/or Dpl related toxicity, cases in which deletion of Bax and overexpression of Bcl-2 confer protection. The mechanism of cell death in infectious PrD may occur through Bax- and Bcl2 -independent pathways, nonapoptotic pathways or another possibility is that if apoptotic pathways are diminished (by deletion of Bax or overexpression of $\mathrm{Bcl}-2$ ) then alternate pathways are rapidly induced. The fact that we observed subtle enhancements of some PrD symptoms may arise from altered brain structure/function in $\mathrm{Bax} \mathrm{KO}$ and $\mathrm{Bcl}-2$ mice, which both possess more neurons because of reduced developmental cell death or because some low level of neuronal apoptosis may be beneficial in prion-inoculated mice perhaps by reducing the production of infectious prions. Our results suggest that therapeutic targeting of Bax- and Bcl-2-dependent apoptotic pathways will not be a viable strategy for combating infectious PrD.

\section{References}

Aguzzi A, Heikenwalder M (2006) Pathogenesis of prion diseases: current status and future outlook. Nat Rev Microbiol 4:765-775.

Caughey B, Baron GS (2006) Prions and their partners in crime. Nature 443:803-810.

Chen DF, Schneider GE, Martinou JC, Tonegawa S (1997) Bcl-2 promotes regeneration of severed axons in mammalian CNS. Nature 385:434-439.

Chiesa R, Piccardo P, Dossena S, Nowoslawski L, Roth KA, Ghetti B, Harris DA (2005) Bax deletion prevents neuronal loss but not neurological symptoms in a transgenic model of inherited prion disease. Proc Natl Acad Sci USA 102:238-243.

Coulpier M, Messiaen S, Hamel R, Fernandez de Marco M, Lilin T, Eloit M (2006) Bax deletion does not protect neurons from BSE-induced death. Neurobiol Dis 23:603-611.

Cronier S, Laude H, Peyrin JM (2004) Prions can infect primary cultured neurons and astrocytes and promote neuronal cell death. Proc Natl Acad Sci USA 101:12271-12276.

Danial NN, Korsmeyer SJ (2004) Cell death: critical control points. Cell 116:205-219.

Dong J, Li A, Yamaguchi N, Sakaguchi S, Harris DA (2007) Doppel induces degeneration of cerebellar Purkinje cells independently of Bax. Am J Pathol 171:599-607.

Dubois-Dauphin M, Frankowski H, Tsujimoto Y, Huarte J, Martinou JC (1994) Neonatal motoneurons overexpressing the bcl-2 protooncogene in transgenic mice are protected from axotomy-induced cell death. Proc Natl Acad Sci USA 91:3309-3313.

Gould TW, Buss RR, Vinsant S, Prevette D, Sun W, Knudson CM, Milligan CE, Oppenheim RW (2006) Complete dissociation of motor neuron death from motor dysfunction by Bax deletion in a mouse model of ALS. J Neurosci 26:8774-8786.

Heitz S, Lutz Y, Rodeau JL, Zanjani H, Gautheron V, Bombarde G, Richard F, Fuchs JP, Vogel MW, Mariani J, Bailly Y (2007) BAX contributes to Doppel-induced apoptosis of prion-protein-deficient Purkinje cells. Dev Neurobiol 67:670-686.

Hetz C, Thielen P, Fisher J, Pasinelli P, Brown RH, Korsmeyer S, Glimcher L (2007) The proapoptotic BCL-2 family member BIM mediates motoneuron loss in a model of amyotrophic lateral sclerosis. Cell Death Differ 14:1386-1389.

Kang SJ, Sanchez I, Jing N, Yuan J (2003) Dissociation between neurodegeneration and caspase-11-mediated activation of caspase-1 and caspase- 3 in a mouse model of amyotrophic lateral sclerosis. J Neurosci 23:5455-5460.

Knudson CM, Tung KS, Tourtellotte WG, Brown GA, Korsmeyer SJ (1995) Bax-deficient mice with lymphoid hyperplasia and male germ cell death. Science 270:96-99.

Kostic V, Jackson-Lewis V, de Bilbao F, Dubois-Dauphin M, Przedborski S (1997) $\mathrm{Bcl}-2$ : prolonging life in a transgenic mouse model of familial amyotrophic lateral sclerosis. Science 277:559-562.

Kristiansen M, Messenger MJ, Klohn PC, Brandner S, Wadsworth JD, Collinge J, Tabrizi SJ (2005) Disease-related prion protein forms aggresomes in neuronal cells leading to caspase activation and apoptosis. J Biol Chem 280:38851-38861.

Li A, Barmada SJ, Roth KA, Harris DA (2007) N-terminally deleted forms of the prion protein activate both Bax-dependent and Bax-independent neurotoxic pathways. J Neurosci 27:852-859.

Liberski PP, Sikorska B, Bratosiewicz-Wasik J, Gajdusek DC, Brown P (2004) Neuronal cell death in transmissible spongiform encephalopathies (prion diseases) revisited: from apoptosis to autophagy. Int J Biochem Cell Biol 36:2473-2490.

Lyahyai J, Bolea R, Serrano C, Monleon E, Moreno C, Osta R, Zaragoza P, Badiola JJ, Martin-Burriel I (2006) Correlation between Bax overexpression and prion deposition in medulla oblongata from natural scrapie without evidence of apoptosis. Acta Neuropathol (Berl) 112:451-460.

Ma J, Wollmann R, Lindquist S (2002) Neurotoxicity and neurodegeneration when PrP accumulates in the cytosol. Science 298:1781-1785.

Martinou JC, Dubois-Dauphin M, Staple JK, Rodriguez I, Frankowski H, Missotten M, Albertini P, Talabot D, Catsicas S, Pietra C, Huarte J (1994) Overexpression of BCL-2 in transgenic mice protects neurons from naturally occurring cell death and experimental ischemia. Neuron 13:1017-1030.

Nicolas O, Gavin R, Braun N, Urena JM, Fontana X, Soriano E, Aguzzi A, Del 
Rio JA (2007) $B c l-2$ overexpression delays caspase- 3 activation and rescues cerebellar degeneration in prion-deficient mice that overexpress amino-terminally truncated prion. FASEB J 21:3107-3117.

Park SK, Choi SI, Jin JK, Choi EK, Kim JI, Carp RI, Kim YS (2000) Differential expression of Bax and $\mathrm{Bcl}-2$ in the brains of hamsters infected with 263K scrapie agent. NeuroReport 11:1677-1682.

Prusiner SB (1998) Prions. Proc Natl Acad Sci USA 95:13363-13383.

Reed JC (2006) Proapoptotic multidomain Bcl-2/Bax-family proteins: mechanisms, physiological roles, and therapeutic opportunities. Cell Death Differ 13:1378-1386.

Sagot Y, Dubois-Dauphin M, Tan SA, de Bilbao F, Aebischer P, Martinou JC, Kato AC (1995) Bcl-2 overexpression prevents motoneuron cell body loss but not axonal degeneration in a mouse model of a neurodegenerative disease. J Neurosci 15:7727-7733.

Selimi F, Vogel MW, Mariani J (2000) Bax inactivation in lurcher mutants rescues cerebellar granule cells but not Purkinje cells or inferior olivary neurons. J Neurosci 20:5339-5345.

Shacka JJ, Roth KA (2006) Bcl-2 family and the central nervous system: from rheostat to real complex. Cell Death Differ 13:1299-1304.

Steele AD, Yi CH (2006) Neuromuscular denervation: Bax up against the wall in amyotrophic lateral sclerosis. J Neurosci 26:12849-12851.

Steele AD, Jackson WS, King OD, Lindquist S (2007) The power of automated high-resolution behavior analysis revealed by its application to mouse models of Huntington's and prion diseases. Proc Natl Acad Sci USA 104:1983-1988.

Tsai MS, Chiu YT, Wang SH, Hsieh-Li HM, Lian WC, Li H (2006) Abolishing Bax-dependent apoptosis shows beneficial effects on spinal muscular atrophy model mice. Mol Ther 13:1149-1155.

Yuan J, Lipinski M, Degterev A (2003) Diversity in the mechanisms of neuronal cell death. Neuron 40:401-413. 